\title{
Photodynamic Therapy and Antitumor Immunity
}

\author{
Sandra O. Gollnick, PhD, PI, PDT \\ PI, PDT Center, Department of Cell Stress Biology; Director, Graduate Studies, Department of \\ Immunology, Roswell Park Cancer Institute, Buffalo, New York
}

\begin{abstract}
Preclinical studies have shown that local photodynamic therapy (PDT) enhances systemic antitumor immunity. In addition, it has long been known that the long-term efficacy of PDT depends on the presence of an intact adaptive immune system. Years of research in the laboratory have attempted to shed light on the mechanisms of the PDT-enhanced antitumor immune response, suggesting that increased expression of proinflammatory cytokines may play a key role. This overview on the immunologic potential of PDT briefly explores these proposed mechanisms and addresses preliminary results with PDT vaccines in combination with surgery as perhaps a new clinical strategy for cancer treatment outside the laboratory.
\end{abstract}

"We can use photodynamic therapy [PDT] to cure a lot of primary tumors, but what about its ability to enhance antitumor immunity?" This question was explored in detail by Sandra O. Gollnick, PhD, of the PDT Center, Department of Cell Stress Biology at Roswell Park Cancer Institute, Buffalo, New York, an immunologist with years of laboratory expertise with PDT. From laboratory findings in mice models to preliminary results in tumor cells in patients, the increased antitumor immune response achieved after PDT continues to be explored. Preclinical mice studies have suggested that through augmenting T-cell proliferation, tumor-infiltrating neutrophils may play a key role in establishing PDTenhanced antitumor immunity. ${ }^{1}$ A clearer understanding of the mechanisms of action regarding this enhancement in antitumor immune response with PDT represents the first step on the road to establishing a new treatment strategy that takes advantage of this phenomenon, which requires further testing in clinical trials.

\section{PDT and Antitumor Immunity: Moving From Mice to Humans}

"Long-term PDT efficacy depends upon the presence of an intact adaptive immune system," declared Dr. Gollnick. Preclinical studies using mice models and clinical studies in patients have shown that PDT is capable of influencing the immune system. ${ }^{2}$ Studies have also shown that response to PDT is limited in immunosuppressed patients, she added. "When we do PDT in immunocompetent BALB/c mice, we get a very good response, but when we do it in immunosuppressed or immunoincompetent mice, we don't get the response," reported Dr. Gollnick. However, the response is restored when CD8+ T cells are given back to the mice, suggesting that PDT-enhanced antitumor immunity may be mediated by CD8+ T cells.

Furthermore, preclinical mice studies have shown a connection between T-cell proliferation and antitumor immunity after PDT. ${ }^{1}$ For instance, a PDT regimen that induces a high level

Copyright $\odot 2012$ by the National Comprehensive Cancer Network. All rights reserved.

Correspondence: Sandra O. Gollnick, PhD, Roswell Park Cancer Institute, Department of Cell Stress Biology, Elm \& Carlton Streets, Buffalo, NY 14263. Sandra.gollnick@ roswellpark.org. 
of neutrophilic infiltrate-generated tumor-specific T-cell responses, whereas a PDT regimen that induces little or no neutrophilic infiltrate exhibited minimal antitumor activity. ${ }^{1}$

"We showed this in animals for a decade and then did it on patients," revealed Dr. Gollnick. The first actual study in humans tested the immune response to PDT in patients with basal cell carcinoma. ${ }^{3}$ "Our laboratory defined an antigen that is overexpressed on basal cell carcinomas Hip1 (a member of the sonic hedgehog pathway)," she explained. The lesions were either treated with PDT or surgically removed. Both before and after PDT, "we saw an increased T-cell response against that Hip1 antigen that we did not see following surgery," Dr. Gollnick reported. This systemic antitumor immune response was inversely correlated with treatment area and dose.

\section{Mechanisms of Action: Antitumor Inflammation and Neutrophil Migration}

The efficacy of PDT appears to be strongly linked to the degree and nature of inflammation induced by PDT. ${ }^{2}$ The induction of adaptive immunity is dependent on this inflammation, explained Dr. Gollnick. PDT induces both acute local and systemic inflammation, which is characterized by 2 steps: 1) the rapid influx of neutrophils into the treated tumor bed, and 2) an increased expression of proinflammatory cytokines ${ }^{4,5}$ (eg, interleukin [IL]-6).

According to Dr. Gollnick et al, ${ }^{4}$ the general inflammatory response to PDT may be mediated, at least partly, by IL-6, which may in turn modulate the local antitumor immune response. In contrast, they also found that enhanced IL-10 expression may play a role in the suppression of cell-mediated responses seen after PDT. ${ }^{4}$ Another group of investigators explored the effects of cytokine overexpression on PDT. ${ }^{5}$ They showed that IL- 6 expression seems to modulate cellular sensitivity to PDT. ${ }^{5}$ Furthermore, tumor oxygenation, extent of tumor damage, and vascular damage may be correlated with induction of inflammation, as measured by IL- 6 and macrophage inflammatory protein 1 and 2 expression. ${ }^{6}$

Moreover, animal models have shown that induction of inflammation, and therefore PDT enhancement of antitumor immunity, is regimen-dependent. ${ }^{1}$ In one study, ${ }^{6}$ animals were treated with high- or low-dose PDT or not treated at all. In the primary response, 2 days after treatment with PDT, an increased antitumor immune response was seen with both regimens. However, 60 days after treatment with PDT, a difference was seen between the regimens: "where you induced the most inflammation, you got the most long-term efficacy," reported Dr. Gollnick.

Research on the effect of GR1+ cells on tumor response to PDT was shared by Dr. Gollnick. This animal model seemed to show a better response with high-dose PDT than with lowdose PDT. At 60 days after treatment, more than $75 \%$ of the mice treated with high-dose PDT were tumor-free, compared with only approximately $30 \%$ of the mice treated with lowdose PDT (Figure 1). "Interestingly, when we deplete those neutrophils in the high-dose PDT, we don't see a big difference. When we deplete them in the low-dose PDT, we saw a big drop off in response," she revealed, concluding that GR1+ cells appear to play an important role in PDT-induced antitumor inflammation.

"Generally, immunologists have never paid attention to neutrophils," joked Dr. Gollnick, until now. "We found that neutrophils were doing more than just coming into the tumor bed to clear the debris; they were also in some way stimulating antitumor immunity," she reasoned. N1 neutrophil migration to tumor-draining lymph nodes (TDLNs) stimulates Tcell activation. ${ }^{1}$ Kousis et $\mathrm{al}^{1}$ found that PDT increases the presence of N1 neutrophils in TDLNs and that N1 neutrophil migration is critical to the generation of effector T cells. 
Further research suggested that IL-17, the hall-mark cytokine of the T helper 17 cell subset, may regulate this migration of neutrophils into inflamed tissue. "When mice were no longer able to respond to IL-17, we decreased the entry of the neutrophils into the TDLNs," explained Dr. Gollnick. IL-17 binds its receptor complex, which is composed of IL-17RA and IL-17RC. ${ }^{7}$ IL-17RA tends to enhance the efficacy of PDT, she added, and IL-17 regulates cytotoxicity in vivo after PDT. "When you look at the TDLN population in the absence of IL-17, you lost T-cell efficacy as well." Therefore, induction of antitumor immunity by PDT seems to depend on both neutrophils and IL-17.

Once these discoveries were made, Dr. Gollnick stated that the focus of her laboratory changed. "Now we worked on models where we could both control primary tumors and enhance antitumor immunity," she stated. Her laboratory treated mice at the low-dose PDT regimen, waited 10 days, and then treated them at the high-dose PDT regimen. With this technique, called split-dose PDT, Dr. Gollnick and her colleagues were able to boost the long-term efficacy of the low-dose regimen much higher than the low-dose regimen alone. The split-dose PDT increased the number of activated tumor-specific T cells (Figure 2), thereby enhancing antitumor immunity.

Based on these research findings, it became clear that PDT combination regimens could be devised both to control and eliminate primary tumors while enhancing antitumor immunity. "We know how we can do this in animals; now we need to talk about how we can do it in humans," Dr. Gollnick proposed.

\section{PDT-Generated Vaccines: On the Road to Clinical Trials}

First, Dr. Gollnick et $\mathrm{al}^{8}$ examined the immunogenicity of PDT-generated murine tumor cell lysates in a preclinical vaccine model. "When we take and treat tumor cells ex vivo, they become very effective vaccines," she revealed. PDT vaccines are tumor-specific and seem to induce a cytotoxic T-cell response. " 8 "Surprisingly, we saw significant improved response when we used PDT lysate." These vaccines seemed to inhibit the growth of both new and established tumors, suggesting their clinical potential as an adjuvant therapy.

The generation of effective vaccines depends on active metabolism, at least 8 hours of protein synthesis, and the ability to stimulate the maturation of dendritic cells, explained Dr. Gollnick. "An important part in terms of an eventual clinical trial is to show the vaccine is efficacious before injecting it into patients," she added. The preclinical steps include treatment of tumor cells with PDT in vitro and incubation of the cells for more than 8 hours after PDT. The clinical steps include disaggregation of surgically removed tumors, treatment with PDT, incubation of treated samples for 18 hours, and irradiation. Vaccination of mice with PDT-generated vaccines increased tumor-specific immunity, as measured through testing for increased cytotoxic T-cell activity, Dr. Gollnick reported.

Dr. Gollnick discussed the preclinical use of PDT-treated tumor cells as an effective adjuvant therapy. PDT vaccines have been studied in combination with surgery to enhance antitumor immunity in aggressive melanoma B16 tumors and SCVII head and neck tumors. In both cases, most of the tumors were surgically removed, treated ex vivo with PDT, and then used to vaccinate mice whose tumors had been removed surgically. Combination treatment offered better protection against the tumor than did surgery alone (Figure 3). "We began to see survival out to 40 days, and now out to 90 days," declared Dr. Gollnick.

In addition, PDT-treated tumor cells have been used in combination with surgery to control metastatic tumor growth of breast carcinoma. These tumors metastasize to the lymph node, brain, and bone by approximately 14 to 18 days, Dr. Gollnick noted. "Surprisingly good results were obtained with split-dose PDT in controlling tumor response long term, and there 
was a great response compared with surgery alone," she added. A clinical trial of PDT vaccine for late-stage melanoma is making its way through the institutional review board.

\section{Conclusions}

Findings from the long hours spent by investigators in research laboratories have shown that PDT enhances antitumor immunity. The induction of this adaptive immunity seems to be dependent on local and systemic inflammation, which is characterized by the rapid influx of neutrophils into the treated tumor bed and an increased expression of proinflammatory cytokines. As more has been learned about the mechanisms of action of this immune response to PDT, combination therapies, including PDT vaccines, are becoming the focus of clinical trials, such as for head and neck tumors and melanoma. "For combination therapies, we have to be smart about how we do it in the clinic," Dr. Gollnick said in closing.

\section{Acknowledgments}

Dr. Gollnick is a Principal Investigator of an NCI-funded grant awarded to Roswell Park Cancer Institute to support studies in photodynamic therapy.

\section{References}

1. Kousis PC, Henderson BW, Maier PG, Gollnick SO. Photodynamic therapy enhancement of antitumor immunity is regulated by neutrophils. Cancer Res. 2007; 67:10501-10510. [PubMed: 17974994]

2. Brackett CM, Gollnick SO. Photodynamic therapy enhancement of anti-tumor immunity. Photochem Photobiol Sci. 2011; 10:649-652. [PubMed: 21253659]

3. Kabingu E, Oseroff AR, Wilding GE, Gollnick SW. Enhanced systemic immune reactivity to a basal cell carcinoma associated antigen following photodynamic therapy. Clin Cancer Res. 2009; 15:4460-4466. [PubMed: 19549769]

4. Gollnick SO, Liu X, Owczarczak B, et al. Altered expression of interleukin 6 and interleukin 10 as a result of photodynamic therapy in vivo. Cancer Res. 1997; 57:3904-3909. [PubMed: 9307269]

5. Usuda J, Okunaka T, Turukawa K, et al. Increased cytotoxic effects of photodynamic therapy in IL-6 gene transfected cells via enhanced apoptosis. Int J Cancer. 2001; 93:475-480. [PubMed: 11477550]

6. Henderson BW, Gollnick SO, Snyder JW, et al. Choice of oxygen-onserving treatment regimen determines the inflammatory response and outcome of photodynamic therapy of tumors. Cancer Res. 2004; 64:2120-2126. [PubMed: 15026352]

7. Gaffen SL. Structure and signaling in the IL-17 receptor family. Nat Rev Immunol. 2009; 9:556567. [PubMed: 19575028]

8. Gollnick SO, Vaughan L, Henderson BW. Generation of effective antitumor vaccines using photodynamic therapy. Cancer Res. 2002; 62:1604-1608. [PubMed: 11912128] 


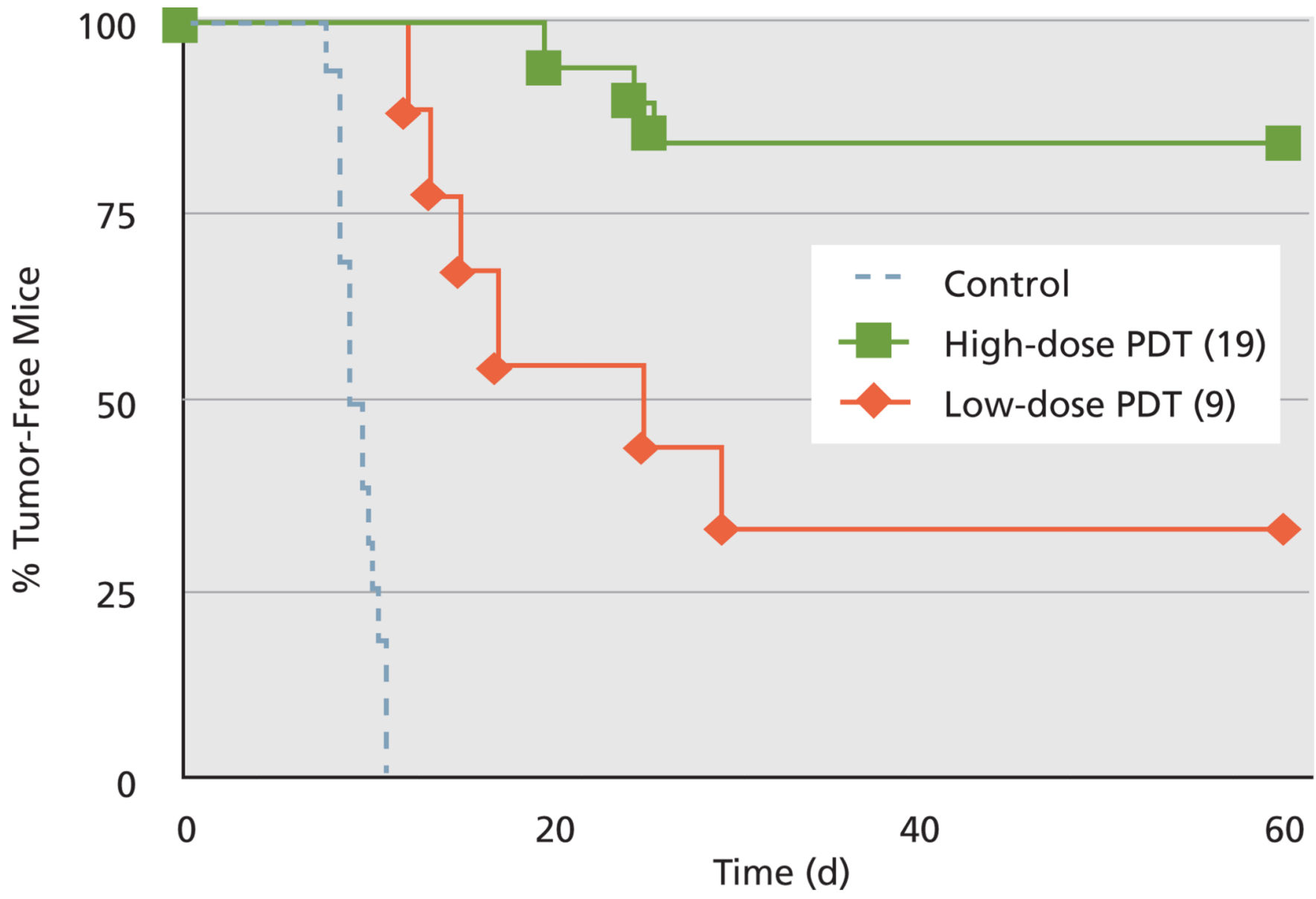

Figure 1.

Effect of GR $1^{+}$cells on tumor response to photodynamic therapy (PDT). By day 60, more mice treated with high-dose PDT were tumor-free than were mice treated with low-dose PDT. 

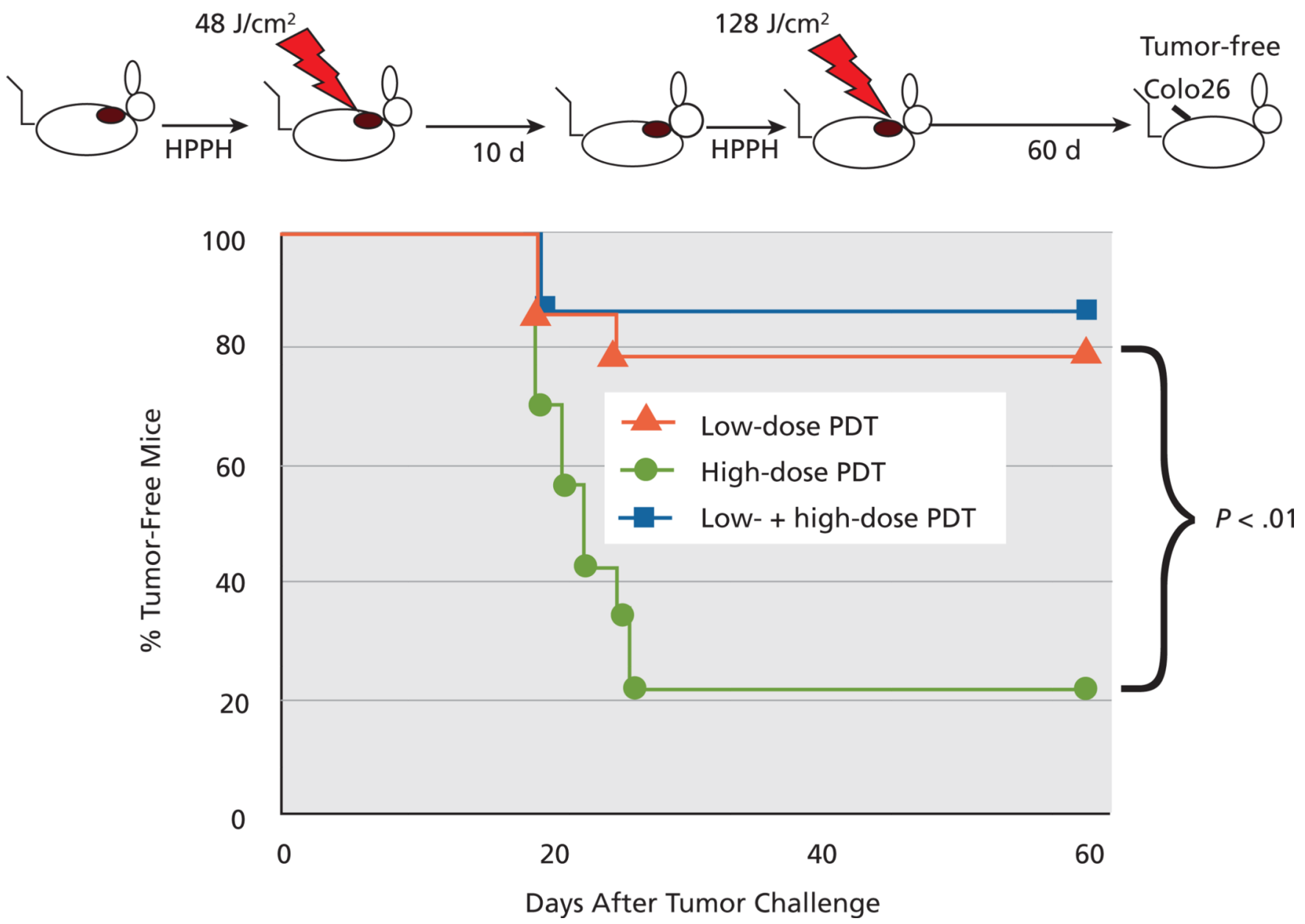

Figure 2.

Enhanced antitumor immunity after split-dose photodynamic therapy (PDT). Sixty days after tumor challenge, more mice treated with split-dose PDT were tumor-free than were mice treated with low-dose and especially high-dose PDT. HPPH, 2-[1-hexyloxyethyl]-2devinyl pyropheophorbide-a. 


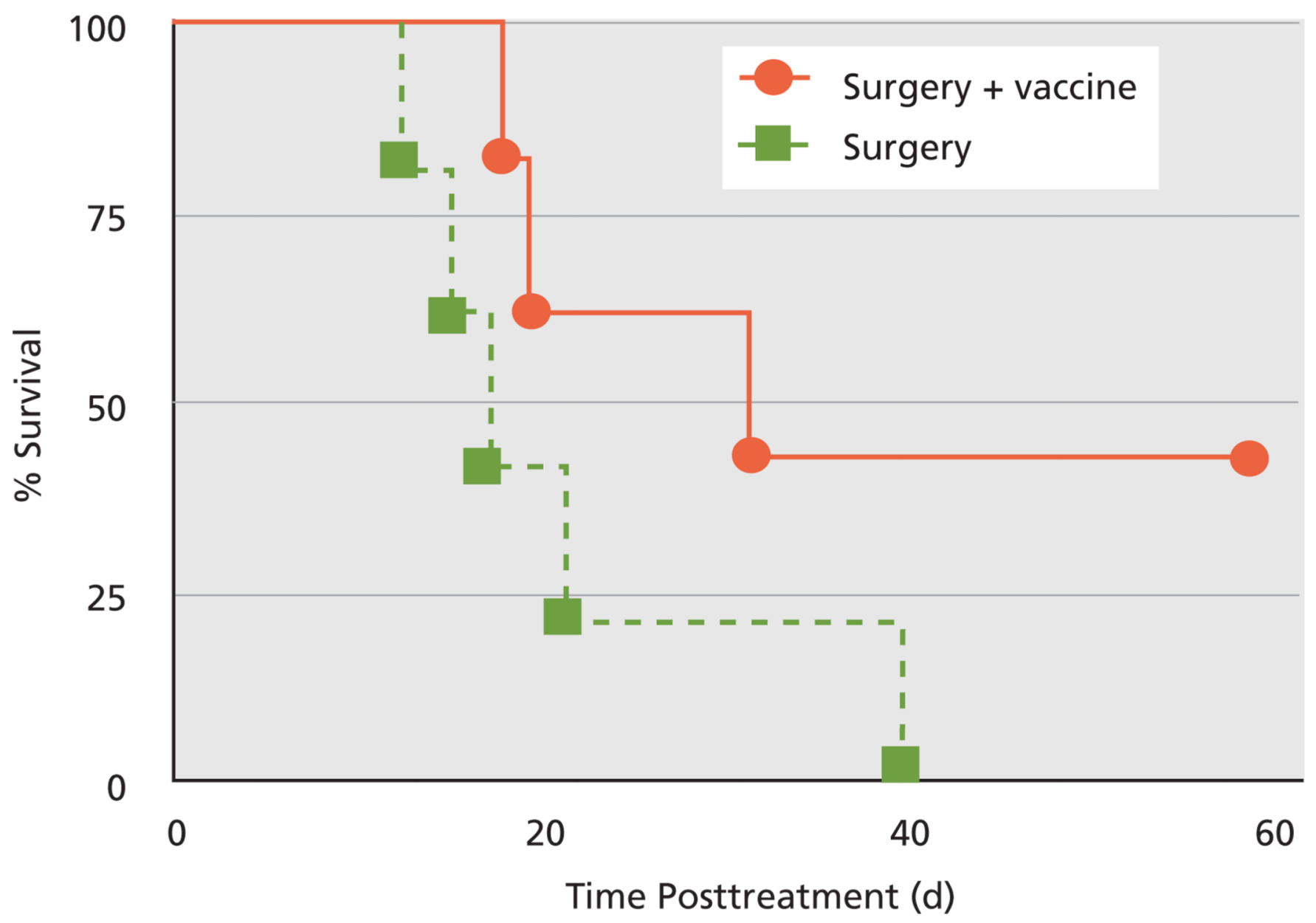

Figure 3.

Photodynamic therapy (PDT) vaccine plus surgery. Mice treated with the combination of PDT vaccine plus surgery lived longer than mice treated with surgery alone. 